\title{
Addressing key issues in photosynthetic sunlight harvesting by utilizing coherence: a perspective
}

\section{Perspective}

Coherence, as embodied by interference effects between waves, be it classical waves or matter waves, is simple to understand in terms of phase differences but often very bewildering to interpret and yet very important in many branches in science and engineering. Just like the superposition of waves, superposition of wave functions corresponding to stationary Eigen states of atoms or molecules can create non-stationary states. If we can lock the phases of these eigenstates initially, the nonstationary state-called a coherent superposition state-subsequently loses its amplitude due to time evolution of each eigenstate leading to out-of-phase oscillations, a process known as dephasing (as well as due to irreversible relaxation of amplitude known as decoherence). Preparing and preserving such a coherent superposition state is crucial for the outcome of a (photo) chemical process ${ }^{1}$ and serves as a lifeline to keep the dream of quantum coherent control and quantum computing alive. Such a state is quite short-lived but, fortunately, can be time-resolved by short bursts of light pulses using a femtosecond pulsed laser.

With the advent of two-dimensional electronic spectroscopy $(2 \mathrm{DES}),{ }^{2}$ the existence of delocalized electronic states or excitonic states extended over few chlorophyll molecules within an aggregate wrapped inside a protein matrix, known as a pigment-protein complex (PPC) was identified ${ }^{3}$ which are manifested as cross-peaks in a 2D spectrum (analogous to the cross-peaks in a 2D NMR spectrum). Moreover, in subsequent experiments, coherent superposition between different excitonic states was found to exist for unusually long time at cryogenic temperature ${ }^{4}$ as well as at room temperature. ${ }^{5}$ In nature, the efficiency of transferring energy of sun light, a process known as excitation energy transfer (EET), was found to be nearly unity for certain photosynthetic bacteria. The coherent EET pathway via energy delocalization (as opposed to incoherent EET via localized hopping of energy) was identified to render this remarkable efficiency where delocalization helps in avoiding a local energy trap and coherent coupling ensures robustness against environmental fluctuations. ${ }^{6,7}$ Long distance coupling between far apart chromophores across a $\sim 5 \mathrm{~nm}$ wide protein was also reported. ${ }^{8}$ It is noteworthy to mention here that a recent report has challenged all these previous experimental observations ${ }^{9}$ but this, by and large, has not been accepted within the community till now.

The first crucial question is: "What is exactly meant by coherence in the context of efficient EET by PPCs in nature?" Often encountered during a (scientific) conversation with an outlander to this field is the question: "What is so greatness about the phenomena with green plants at low temperature?" that invariably comes with a comment: "So, this is nothing but doing the double-slit experiment in low temperature to enhance signal-to-noise!" The one-line answer to this trivial question is that the coherence during EET is a (non-linear or higher-order) coherence between the excited (vibronic) states and not a (linear or first-order) coherence between ground and excited states (i.e. minimum two field interactions are required to create the
Volume I Issue I - 2017

\author{
Arijit K De \\ Department of Chemical Sciences, Indian Institute of Science \\ Education and Research, India
}

Correspondence: Arijit K De, Department of Chemical Sciences, Indian Institute of Science Education and Research (IISER) Mohali, Knowledge City, Sector 8I, SAS Nagar, Punjab 140 306, India,

Email arijitb4u@gmail.com; akde@iisermohali.ac.in

Received: August 04, 2017| Published:September 06, 2017

initial superposition states as opposed to just one field interaction). However, there are some major non-trivial issues that I would like to address here.

The coherent EET model was greatly challenged by the fact that the pioneering experiments were carried out in cryogenic temperature under laser excitation (as a source of coherent excitation), conditions that are far from real physiological conditions in elevated temperature under (incoherent) sun light excitation. ${ }^{9}$ This issue can be satisfactorily answered by arguing that:

i. Although a laser pulse prepares the initial coherent superposition state, its subsequent time evolution is solely governed by the motions of a PPC only (i.e. by the field-free system Hamiltonian) which ensures that the coherence is preserved for sufficiently long time by which the energy leaves the PPC.

ii. Although sunlight is a collection of 'statistically' incoherent photons, every single photon-single PPC interaction is very much coherent in nature (such weak measurement on ultrafast time scale has not been attempted till date!).

Although the existence, importance and, to a great extent, the exact nature (vibronic, as opposed to purely electronic, ${ }^{10}$ of coherence has been well explored, the exact biological role of coherence is yet to be underpinned: "Is coherence a byproduct or is it absolutely necessary for efficient EET within PPCs? If so, is it a sufficient condition? Or adapting to a fluctuating 'noisy' thermal environment is a more crucial issue?" Note that while this energy delocalization and coherent coupling may be necessary for robustness, the energy has to eventually migrate; so, a more involved question is: "Does this noise assist in EET?"11 Even if we assume that coherence is absolutely important, at least within PPCs adapted to low sunlight condition (for example, within the FMO complex of green sulfur bacteria), there still remains few more questions: "To what extent it is important for PPCs encountered in terrestrial plans that get sufficient (and, at times, excess) sun light?" Finally, another crucial but yet to be resolved issue is related to the actual time scale of coherent energy 
transfer for a single PPC, without averaging over an ensemble of PPCs, to accurately measure the pure dephasing rate which is often obscured by fast ensemble dephasing rates. ${ }^{12}$ For such ultra-sensitive measurements, fluorescence-detected 2DES has been proposed. ${ }^{13}$

The ultimate wisdom about the functioning of a PPC lies in the answers to these key questions.

\section{Acknowledgments}

The author sincerely acknowledges IISER Mohali for providing start-up grant.

\section{Conflicts of interest}

There is no conflict of interest.

\section{References}

1. ScholesGD,FlemingGR, ChenLX, etal.Usingcoherencetoenhancefunction in chemical and biophysical systems. Nature. 2017;543(7647):647-656.

2. Arijit K De. Conceptual understanding of optical multi-dimensional coherent spectroscopy. Journal of Postdoctoral Research 2014;2(7):23-28

3. Brixner T, Stenger J, Vaswani HM, et al. Two-dimensional spectroscopy of electronic couplings in photosynthesis. Nature. 2005;434(7033):625-628.

4. Engel GS, Calhoun TR, Read EL, et al. Evidence for wavelike energy transfer through quantum coherence in photosynthetic systems. Nature. 2007;446(7137):782-786.

5. Panitchayangkoon G, Hayes D, Fransted KA, et al. Long-lived quantum coherence in photosynthetic complexes at physiological temperature. Proc Natl Acad Sci USA. 2010;107(29):12766-12770.
6. Collini E, Wong CY, Wilk KE, et al. Coherently wired light-harvesting in photosynthetic marine algae at ambient temperature. Nature. 2010;463(7281):644-647.

7. Ishizaki A, Fleming GR. Quantum Coherence in Photosynthetic Light Harvesting. Annu Rev Condens Matter Phys. 2012;3:333-361.

8. Duan HG, Prokhorenko VI, Cogdell RJ, et al. Nature does not rely on long-lived electronic quantum coherence for photosynthetic energy transfer. Proc Natl Acad Sci USA. 2017;114(32):8493-8498.

9. Brumer P, Shapiro M. Molecular response in one-photon absorption via natural thermal light vs. pulsed laser excitation. Proc Natl Acad Sci USA. 2012;109(48):19575-19578.

10. Romero E, Augulis R, Novoderezhkin VI, et al. (2014) Quantum coherence in photosynthesis for efficient solar-energy conversion. Nat Phys. 2014;10(9):676-682.

11. Caruso F, Alex WC, Animesh D, et al. Highly efficient energy excitation transfer in light-harvesting complexes: The fundamental role of noiseassisted transport. Quantum Physics. 2009;131:105106.

12. Dawlaty JM, Ishizaki A, De AK, et al. Microscopic quantum coherence in a photosynthetic light-harvesting antenna. Philos Trans A Math Phys Eng Sci. 2012;370(1972):3672-3691.

13. De AK, Monahan D, Dawlaty JM, et al. Two-dimensional fluorescencedetected coherent spectroscopy with absolute phasing by confocal imaging of a dynamic grating and 27-step phase-cycling. $J$ Chem Phys. 2014;140(19):194201. 